\title{
Tribbles homologue 3 stimulates canonical TGF- $\beta$ signalling to regulate fibroblast activation and tissue fibrosis
}

\author{
Michal Tomcik, ${ }^{1,2}$ Katrin Palumbo-Zerr, ${ }^{1}$ Pawel Zerr, ${ }^{1}$ Barbora Sumova, ${ }^{1,2}$ \\ Jerome Avouac, ${ }^{3}$ Clara Dees, ${ }^{1}$ Alfiya Distler, ${ }^{1}$ Radim Becvar, ${ }^{2}$ Oliver Distler, ${ }^{4}$ \\ Georg Schett, ${ }^{1}$ Ladislav Senolt, ${ }^{2}$ Jörg H W Distler ${ }^{1}$
}

\begin{abstract}
Handling editor Tore K Kvien
- Additional material is

published online only. To view please visit the journal online (http://dx.doi.org/10.1136/ annrheumdis-2014-206234).

${ }^{1}$ Department of Internal Medicine III and Institute for Clinical Immunology, University of Erlangen-Nuremberg, Erlangen, Germany

${ }^{2}$ Department of Rheumatology, 1st Faculty of Medicine, Institute of Rheumatology, Charles University, Prague, Czech Republic

${ }^{3}$ Rheumatology A Department, Paris Descartes University, Cochin Hospital, Paris, France

${ }^{4}$ Center of Experimental Rheumatology and Zurich Center of Integrative Human Physiology, University Hospital, Zurich, Switzerland
\end{abstract}

\section{Correspondence to}

Dr Jörg H W Distler, Department of Internal Medicine 3, Institute for Clinical Immunology, University of Erlangen-Nuremberg, Erlangen D-91054, Germany; joerg.distler@uk-erlangen.de

Received 8 July 2014 Revised 29 December 2014 Accepted 6 January 2015 Published Online First 20 January 2015

CrossMark

\section{To cite: Tomcik M,} Palumbo-Zerr K, Zerr $\mathrm{P}$ et al. Ann Rheum Dis 2016;75:609-616.

\section{ABSTRACT}

Objectives Tribbles homologue 3 (TRB3) is a pseudokinase that modifies the activation of various intracellular signalling pathways to control fundamental processes extending from mitosis and cell activation to apoptosis and modulation of gene expression. Here, we aimed to analyse the role of TRB3 in fibroblast activation in systemic sclerosis (SSc).

Methods The expression of TRB3 was quantified by quantitative $P C R$, western blot and

immunohistochemistry. The role of TRB3 was analysed in cultured fibroblasts and in experimental fibrosis using small interfering RNA (siRNA)-mediated knockdown and overexpression of TRB3.

Results TRB3 expression was increased in fibroblasts of patients with SSC and in murine models of SSC in a transforming growth factor- $\beta$ (TGF- $\beta$ )/Smad-dependent manner. Overexpression of TRB3 stimulated canonical TGF- $\beta$ signalling and induced an activated phenotype in resting fibroblasts. In contrast, knockdown of TRB3 reduced the profibrotic effects of TGF- $\beta$ and decreased the collagen synthesis. Moreover, siRNA-mediated knockdown of TRB3 exerted potent antifibrotic effects and ameliorated bleomycin as well as constitutively active TGF- $\beta$ receptor I-induced fibrosis with reduced dermal thickening, decreased hydroxyproline content and impaired myofibroblast differentiation.

Conclusions The present study characterises TRB3 as a novel profibrotic mediator in SSc. TGF- $\beta$ induces TRB3, which in turn activates canonical TGF- $\beta /$ Smad signalling and stimulates the release of collagen, thereby inducing a positive feedback loop that may contribute to aberrant TGF- $\beta$ signalling in SSC.

\section{INTRODUCTION}

Systemic sclerosis (SSc) is a prototypical multisystem fibrotic disorder that is considered as one of the most challenging rheumatic diseases. During the course of the disease, progressive accumulation of extracellular matrix disrupts the physiological tissue architecture and causes organ failure, resulting in high morbidity and mortality among patients with SSc. ${ }^{1}$ The molecular mechanisms underlying the aberrant activation of fibroblasts in SSc are incompletely understood. Transforming growth factor- $\beta$ (TGF- $\beta$ ) potently stimulates the release of collagen from fibroblasts, and prolonged TGF- $\beta$ signalling has been proposed as a key mechanism in the pathogenesis of SSc and other fibrotic diseases. ${ }^{2}$ In contrast to physiological wound healing, where TGF- $\beta$ signalling is terminated after completion of wound repair, TGF- $\beta$ signalling remains persistently activated in SSc with increased nuclear accumulation of Smad3 and increased transcription of TGF- $\beta$ target genes in fibroblasts. ${ }^{3}$ However, it remains elusive to date, why termination of TGF- $\beta$ signalling in SSc does not occur. ${ }^{2}$

Tribbles homologue 3 (TRB3, also known as TRIB3, NIPK and SKIP3) is the mammalian homologue of Drosophila Tribbles. ${ }^{4}$ TRB3 is a pseudokinase, which contains a Ser/Thr protein kinase-like domain, but lacks the ATP-binding pocket and catalytic residues. ${ }^{5}$ As TRB3 lacks kinase activity, it does not directly regulate gene transcription, but exerts its cellular effects indirectly by serving as a molecular scaffold for the assembly of coactivator and corepressor complexes. ${ }^{6}$ TRB3 has been demonstrated to interact with several transcriptional mediators such as CCAAT-enhancer-binding protein homologous protein (CHOP), peroxisome proliferator-activated receptor alpha (PPAR $\alpha$ ), activating transcription factor 4 (ATF4) and mothers against decapentaplegic homolog 3 (Smad3). ${ }^{7-9}$ Through those interactions, TRB3 coordinates crucial cellular processes such as glucose and lipid metabolism, ${ }^{7} 10$ adipocyte differentiation $^{11}$ or apoptosis. ${ }^{8}$ TRB3 has also recently been implicated in the pathogenesis of osteoarthritis. TRB3 is upregulated in osteoarthritic chondrocytes and inhibits cell survival and proteoglycan synthesis. ${ }^{12}$

Here, we aimed to characterise the role of TRB3 in fibrosis. Using functional in vitro studies and complementary mouse models of SSc, we demonstrate that TRB3 regulates fibroblast activation in SSc. TRB3 is induced by TGF- $\beta$ in SSc and in turn stimulates TGF- $\beta /$ Smad signalling in SSc fibroblasts. Knockdown of TRB3 interrupts this positive feedback loop and exerts potent antifibrotic effects in cultured fibroblasts and in bleomycin as well as TGF- $\beta$ receptor I (T $\beta$ RI)-induced skin fibrosis.

\section{MATERIALS AND METHODS}

\section{Patients and fibroblast cultures}

Dermal fibroblasts were isolated from skin biopsies of patients with SSc $(n=20)$ and from age-matched and sex-matched healthy volunteers $(n=20) .{ }^{13}{ }^{14}$ All patients with SSc fulfilled the criteria for SSc as suggested by LeRoy and Medsger ${ }^{15}$ as well as the 
American College of Rheumatology/European League Against Rheumatism 2013 criteria. ${ }^{16}$ Sixteen patients were women, four were men. Ten patients had limited cutaneous SSc and 10 diffuse cutaneous SSc. The median age of patients with SSc was 49 years (range 19-68 years), and their median disease duration (from the onset of the first non-Raynaud's symptom attributable to SSc) was 5 years (range $0.5-12$ years). None of the patients were receiving disease-modifying antirheumatic drugs or glucocorticoids at the time of biopsy. All patients and control subjects signed a consent form approved by the local institutional review board. Fibroblasts from passages 4 to 8 were used for the experiments.

\section{Cloning of human TRB3 gene}

The sequences of the primer pairs used for the amplification of the human TRB3 gene are shown in the online supplementary table S1. Truncation constructs with deletion of $180-315$ bp of the wild-type TRB3 sequence was created by site-directed mutagenesis. The sequences of the primer pairs used are shown in the online supplementary table S1. The PCR products were purified by electrophoresis, digested with Bam HI and XhoI (New England Biolabs, Ipswich, Massachusetts, USA) and ligated into a pcDNA3.1(+) expression vector (Life Technologies, Darmstadt, Germany). The expression constructs were amplified in Escherichia coli Dh5 $\alpha$ bacteria (Novagen, Darmstadt, Germany). The expression constructs were commercially sequenced to confirm the identity, integrity and absence of PCR-introduced mutations in the cloned gene.

\section{Overexpression and knockdown experiments}

For TRB3 overexpression experiments, $5 \mu \mathrm{g}$ of the plasmid constructs was transfected in dermal fibroblasts using the 4D-Nucleofector (Lonza, Cologne, Germany). ${ }^{17-19}$ Gene silencing was achieved by transfection of $3 \mu \mathrm{g}$ predesigned small interfering RNA (siRNA) duplexes against TRB3 and Smad3 (both from Thermo Scientific, Bonn, Germany) again using the nucleofection technique. Empty pcDNA3.1(+) vectors or vectors with truncated TRB3 constructs and non-targeting siRNAs (Life Technologies, Darmstadt, Germany) served as controls. The sequences of the used siRNAs are shown in the online supplementary table S1.

\section{Reporter assays}

Cells were transfected with the -353 COL1A2-Luc construct or a common pSv- $\beta$-galctosidase control vector $(\beta$-gal) as a transfection control. The -353 COL1A2-Luc construct was kindly provided by M. Trojanowska. In other experiments, cells were transduced with an Ad-CAGA-Luc construct or AdLacZ (MOI 1000). ${ }^{20-23}$ Luciferase activity was determined after $24 \mathrm{~h}$ using a microplate luminometer (Berthold Technologies, Bad Herrenalb, Germany).

\section{Stimulation with TGF- $\beta$ and incubation with the selective TRRI inhibitor SD-208}

In selective experiments, cells were incubated with recombinant TGF- $\beta$ (10 ng/mL; PeproTech, Hamburg, Germany) or the selective T $\beta$ RI inhibitor SD-208. ${ }^{24}$

\section{Quantitative real-time PCR}

Gene expression was quantified by SYBR Green real-time PCR using an ABI Prism 7300 Sequence Detection System (Life Technologies, Darmstadt, Germany). The sequences of the used human and murine primers are shown in the online supplementary table S1. $\beta$-actin was used to normalise for the amounts of loaded complementary DNA. Dissociation curve analysis and samples without enzyme in reverse transcription (nonreverse transcription controls) were used as negative controls.
Differences were calculated with the threshold cycle $(\mathrm{Ct})$ and the comparative $\mathrm{Ct}$ method for relative quantification. ${ }^{25-27}$

\section{Western blot analysis}

Proteins were separated by sodium dodecyl sulfate-polyacrylamide gel electrophoresis and transferred to a polyvinylidene difluoride membrane. The membrane was incubated overnight at $4^{\circ} \mathrm{C}$ with goat anti-p-Smad3 antibodies (Santa Cruz Biotechnology, Heidelberg, Germany) or rabbit anti-TRB3 antibodies (Abcam, UK) and horseradish peroxidase-conjugated secondary antibodies (Dako, Glostrup, Denmark). Blots were visualised by ECL. $\beta$-actin antibody was used as loading control. ${ }^{28} 29$

\section{Immunohistochemistry and immunofluorescence staining}

Skin sections and dermal fibroblasts were stained with rabbit anti-TRB3 antibodies (Abcam, UK), mouse anti- $\alpha$-smooth muscle actin antibodies (clone 1A4; Sigma-Aldrich, Steinheim, Germany), anti-prolyl-4-hydroxylase- $\beta$ antibodies (Acris Antibodies, Herford, Germany) or goat anti-p-Smad2/3 antibodies (Santa Cruz Biotechnology). Horseradish peroxide (HRP)-conjugated or Alexa Fluor antibodies (Life Technologies, Darmstadt, Germany) were used as secondary antibodies. Irrelevant isotype-matched antibodies served as controls. ${ }^{30}{ }^{31}$ Nuclei were stained using 4',6-diamidino-2-phenylindole (Santa Cruz Biotechnology, Heidelberg, Germany). Stainings were visualised using a Nikon Eclipse 80i microscope (Nikon, Badhoevedorp, Netherlands). The fluorescence intensity was quantified using ImageJ software V.1.44.

\section{Quantification of soluble collagen protein}

The amount of soluble collagen in cell culture supernatants was quantified using the SirCol collagen assay (Biocolor, Belfast, Northern Ireland). ${ }^{32}$

\section{In vivo transfection with siRNAs against TRB3}

Complexes of equal volume of siRNA $(10 \mu \mathrm{mol} / \mathrm{L})$ and atelocollagen (Koken, Tokyo, Japan) were prepared as previously described. $^{33} 34$ The used siRNA duplexes (from Thermo Scientific, Bonn, Germany) are shown in online supplementary table S1. Non-targeting siRNA duplexes served as controls. After confirmation of an effective knockdown of TRB3 in vitro, atelocollagen/siRNA complexes $(100 \mu \mathrm{L})$ were injected intracutaneously once weekly.

\section{Mouse models of fibrosis}

Bleomycin-induced skin fibrosis and skin fibrosis induced by adenoviral overexpression of a constitutively active TGF- $\beta$ receptor I (AdTBR) were performed as described. ${ }^{31}$ 35-37 Treatment with siRNA was initiated together with the injections of bleomycin or AdTBR, respectively.

\section{Analysis of murine skin}

Dermal thickness, myofibroblasts counts and hydroxyproline content were analysed as described previously. ${ }^{21} 32 \quad 38-42$

\section{Statistics}

All data are presented as median with IQR, and differences between the groups were tested for their statistical significance by non-parametric Mann-Whitney $U$ test. $p$ Values are expressed as follows: NS, not significant; $0.05>\mathrm{p}>0.01$ as *; $0.01>\mathrm{p}>0.001$ as $* *$ and $\mathrm{p}<0.001$ as $* * *$.

\section{RESULTS}

The expression of TRB3 is increased in SSc

To investigate the role of TRB3 in the pathogenesis of SSc, we first analysed the expression of TRB3. Both mRNA and protein levels of 
TRB3 were increased in the skin of patients with SSc as compared with matched healthy individuals (figure 1A, B). In healthy volunteers, TRB3 was detected at low levels by immunohistochemistry and was mainly restricted to the basal layer of epidermis. In contrast, prominent staining for TRB3 was detected in spindle-shaped cells in the dermis of patients with SSc (figure 1B). Double staining with the fibroblast marker prolyl-4-hydroxylase- $\beta$ confirmed the overexpression of TRB3 in SSc fibroblasts and demonstrated that virtually all SSc fibroblasts express TRB3 (figure 1B). The intensity of the TRB3 staining in the epidermis did not differ between healthy volunteers and patients with SSc. In addition to fibroblasts and cells of the basal layers of epidermis, endothelial cells also stained positive in patients with SSc, but not in controls (figure 1B). The overexpression of TRB3 persisted in cultured SSc fibroblasts, which maintained increased basal mRNA and protein levels of TRB3 even after eight passages in culture (figure 1C, D). We also observed overexpression of TRB3 in bleomycin-induced skin fibrosis with increased mRNA and protein levels of TRB3 in the skin of mice challenged with bleomycin as compared with $\mathrm{NaCl}$-injected control mice (figure 1E, F).

\section{TGF- $\boldsymbol{\beta}$ induces the expression of TRB3 in a Smad3-dependent manner}

TGF- $\beta$ signalling is a common feature of fibrotic diseases and has been shown to contribute to the activated phenotype of cultured SSc fibroblasts. We, therefore, hypothesised that TGF- $\beta$ signalling may stimulate the expression of TRB3. Indeed, the expression of TRB3 in SSc skin colocalised with $\mathrm{p}-\mathrm{Smad} 2 / 3$, a marker of activated canonical TGF- $\beta$ signalling (figure $2 \mathrm{~A}$ ). Similarly, TRB3 colocalised with $\mathrm{p}-\mathrm{Smad} 2 / 3$ in cultured fibroblasts stimulated with TGF- $\beta$ (figure 2B). Moreover, incubation of fibroblasts of healthy individuals with TGF- $\beta$ increased the $\mathrm{mRNA}$ and protein levels of TRB3 (figure 2C, D). Two peaks of TRB3 expression were detected in fibroblasts stimulated with TGF- $\beta$; an early peak after $1 \mathrm{~h}$ for RNA and $4 \mathrm{~h}$ for protein and a second peak after $12 \mathrm{~h}$ and $24 \mathrm{~h}$ (see online supplementary figure S1A, B). The induction of TRB3 by TGF- $\beta$ was dependent on canonical TGF- $\beta / S m a d$ signalling, and knockdown of Smad3 completely abrogated the stimulatory effects of TGF- $\beta$ on TRB3 expression (figure $2 \mathrm{C}, \mathrm{D}$ ). The stimulatory effects of TGF- $\beta$ signalling on TRB3 were confirmed in vivo. Activation of TGF- $\beta$ signalling by overexpression of a constitutively active T $\beta R I$ upregulated the expression of TRB3 in murine skin both on mRNA and protein levels (figure 2E, F).

\section{TRB3 stimulates canonical TGF- $\beta$ signalling and regulates fibroblast activation}

To analyse the functional consequences of the upregulation of TRB3 in SSc fibroblasts, we overexpressed TRB3 in cultured fibroblasts of healthy individuals (see online supplementary figure S2A, B). Overexpression of TRB3 activated canonical TGF- $\beta /$ Smad signalling in fibroblasts and increased the activity in Smad binding element (SBE, CAGA) reporter assays, promoted accumulation of $\mathrm{p}-\mathrm{Smad} 3$ and upregulation of classical TGF- $\beta /$ Smad target genes such as plasminogen activator inhibitor-1 (PAI-1) and connective tissue growth factor (CTGF) (figure 3A-C). Overexpression of TRB3 also stimulated the collagen synthesis as
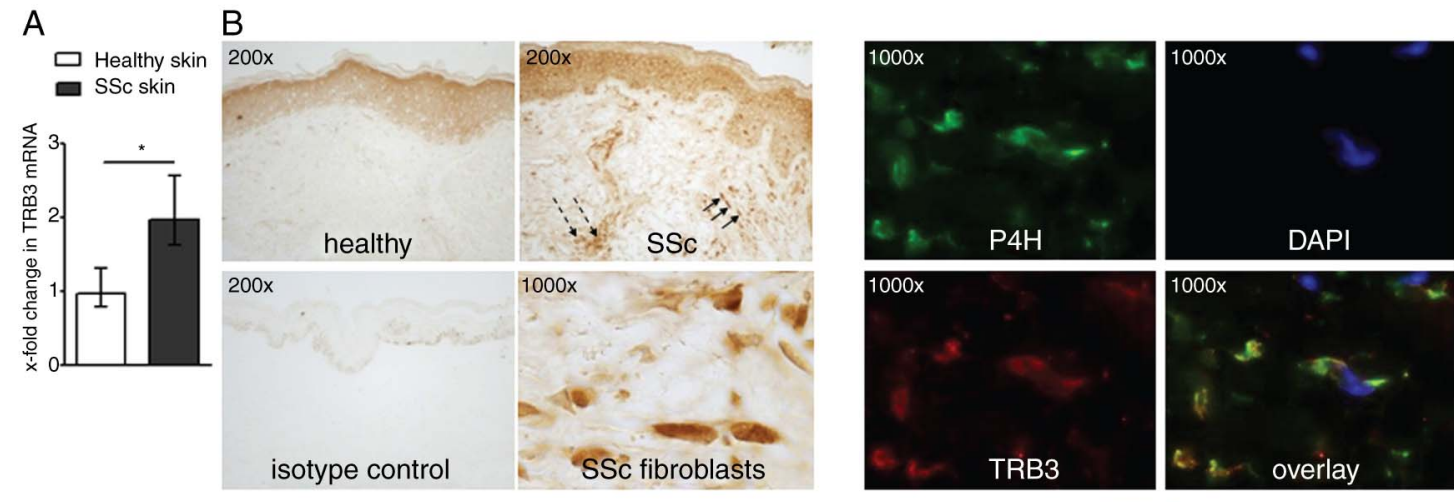

C

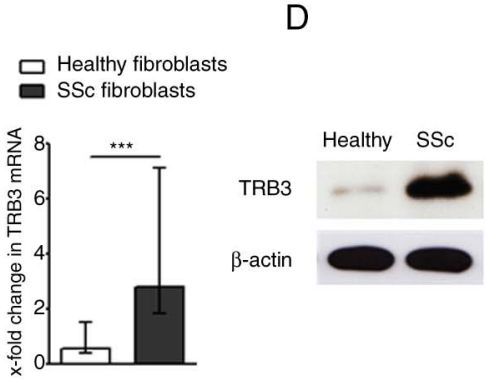

$\mathrm{F}$

$E$

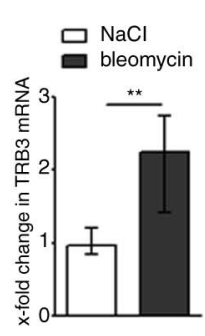

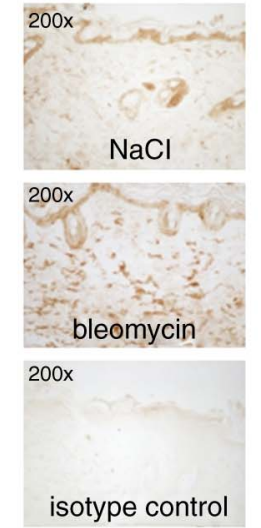

Figure 1 Tribbles homologue 3 (TRB3) is overexpressed in systemic sclerosis (SSc) and in experimental skin fibrosis. (A) The mRNA levels of TRB3 are increased in SSc skin as compared with healthy skin $(n=6)$. (B) Overexpression of TRB3 in the skin of patients with SSC as compared with healthy controls $(n=12)$. The full arrows point at (exemplary) fibroblasts and the interrupted arrows highlight vessels. TRB3 expression in SSc skin colocalises with the fibroblast marker prolyl-4-hydroxylase- $\beta$ (P4H). Nuclei were stained with 4',6-diamidino-2-phenylindole (DAPI). Representative images are shown at 200-fold and 1000-fold magnification. (C and D) The increased expression of TRB3 persists in cultured SSC fibroblasts both on mRNA $(C ; n=6)$ and protein levels $(D ; n=4)$. ( $E$ and $F)$ The mRNA $(E ; n=6)$ and protein $(F ; n=5)$ levels of TRB3 are increased in the skin of mice challenged with bleomycin as compared with NaCl-injected controls. ${ }^{*} 0.05>p>0.01 ;{ }^{* *} 0.01>p>0.001$ and ${ }^{* * *} p<0.001$. 


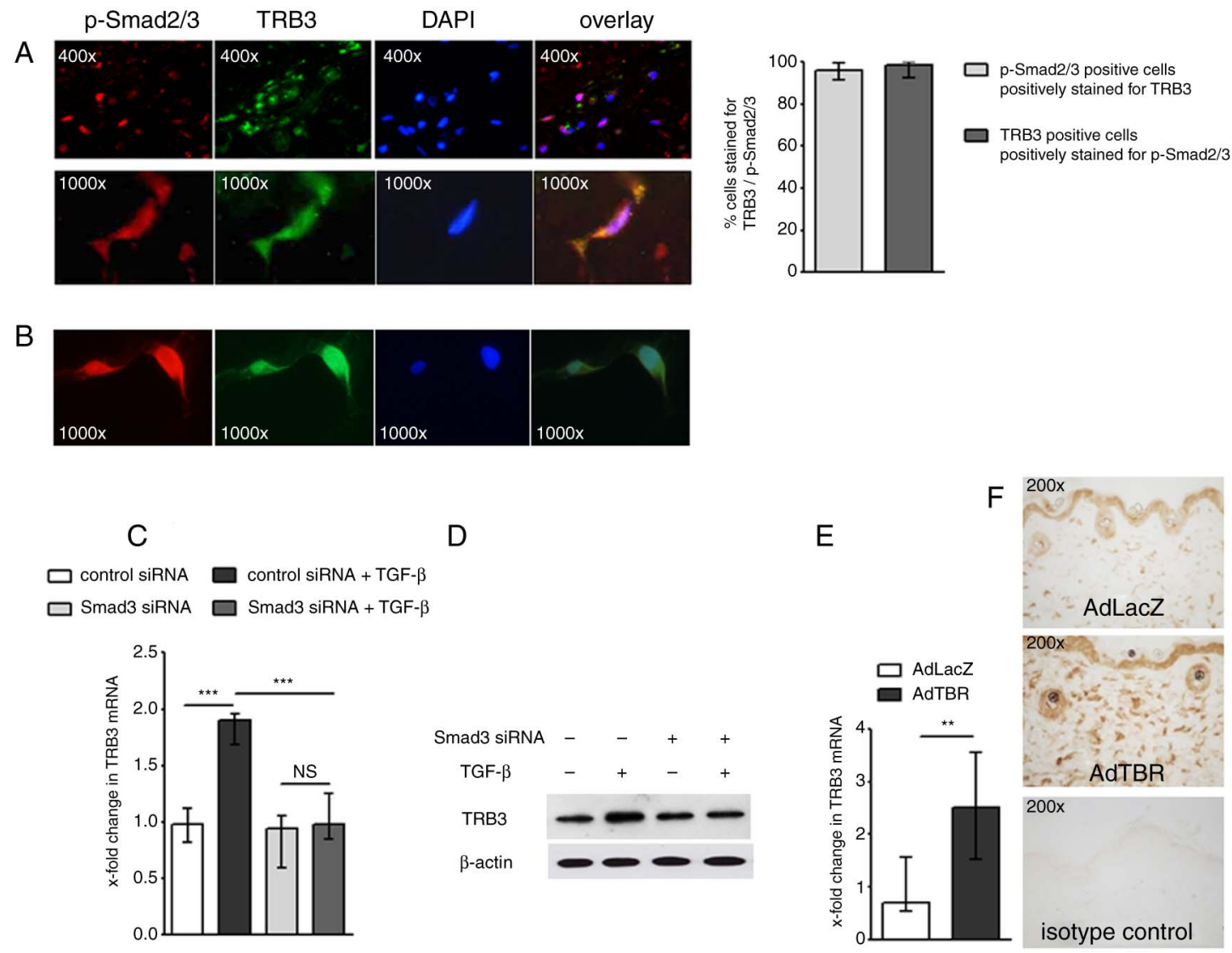

Figure 2 Transforming growth factor- $\beta$ (TGF- $\beta$ ) induces Tribbles homologue 3 (TRB3) in a Smad3-dependent manner. (A and B) TRB3 expression in systemic sclerosis (SSc) skin (A) and in cultured fibroblasts stimulated with TGF- $\beta$ (B) colocalises with phosphorylated Smad2/3 (p-Smad2/3). Nuclei were stained with 4',6-diamidino-2-phenylindole (DAPI). Representative images are shown at 400-fold and 1000-fold magnification. Quantitative analyses demonstrate that almost all TRB3 expressing cells are positive for $\mathrm{p}$-Smad2/3 and vice versa. (C and D) Stimulation of healthy dermal fibroblasts with TGF- $\beta$ increases the mRNA $(C ; n=6)$ and protein level of TRB3 $(D ; n=4)$, an effect that is completely prevented by knockdown of Smad3. (E and F) The mRNA ( $E ; n=6)$ and protein $(F ; n=5)$ levels of TRB3 are induced in murine skin by overexpression of TGF- $\beta$ receptor I (T $\beta R$; adenoviral overexpression of a constitutively active TGF- $\beta$ receptor I (AdTBR)) as compared with AdLacZ controls. NS, not significant; ${ }^{* *} 0.01>p>0.001 ;{ }^{* * *} p<0.001$.

shown by enhanced activity of COL1A2 reporter assays, elevated mRNA levels of COL1A1 and COL1A2 and an increased release of collagen protein (figure 3D-F). Of note, the stimulatory effects of TBR3 on canonical TGF- $\beta$ signalling and on fibroblast activation were comparable with those of high concentrations of recombinant TGF- $\beta$. However, when Smad3 was knocked down by siRNA in fibroblasts overexpressing TRB3, the stimulatory effects of TRB3 overexpression on canonical TGF- $\beta$ signalling and collagen production were completely abrogated (see online supplementary figure $\mathrm{S} 3 \mathrm{~A}-\mathrm{E}$ ), thus highlighting that TRB3 activates TGF- $\beta$ signalling in a Smad3-dependent manner.

To investigate whether TRB3 is required for the stimulatory effects of TGF- $\beta$ on fibroblasts, we inactivated TRB3 in fibroblasts of healthy individuals by siRNA (see online supplementary figure S4A, B). Knockdown of TRB3 did not reduce the expression levels of TGF- $\beta / S$ mad target genes or the basal collagen synthesis in resting healthy fibroblasts. However, in healthy fibroblasts incubated with recombinant TGF- $\beta$, knockdown of TRB3 completely abrogated the TGF- $\beta$-induced SBE reporter activity, the accumulation of phosphorylated Smad3 and the induction of the target genes PAI-1 and CTGF (figure 4A-C). Inactivation of TRB3 also prevented the stimulatory effects of TGF- $\beta$ on collagen synthesis with reduced activation of COL1A2 reporters, decreased levels of COL1A1 and COL1A2
mRNA and reduced collagen content in the supernatants as compared with fibroblasts transfected with non-targeting siRNA (figure 4D-F). In addition, knockdown of TRB3 in dermal fibroblasts of patients with SSc reduced the levels of phosphorylated Smad3 and the basal collagen synthesis as did incubation with the selective T $\beta R I$ inhibitor SD-208 (see online supplementary figure S5A-E).

\section{Knockdown of TRB3 protects from T $\beta R I$-induced fibrosis}

After demonstrating that knockdown of TRB3 inhibits the profibrotic effects of TGF- $\beta$ in vitro, we next aimed to confirm these findings in vivo. We thus inactivated TRB3 by siRNA in the skin of mice overexpressing a constitutively active TGF $\beta$ receptor type I. In vivo transfection with siRNAs against TRB3 effectively decreased the mRNA and protein levels of TRB3 in murine skin (see online supplementary figure S6A, B). Inactivation of TRB3 exerted potent antifibrotic effects (figure 5A-E). Knockdown of TRB3 strongly reduced dermal thickening compared with AdTBR mice injected with non-targeting siRNA $(p<0.05)$ (figure $5 \mathrm{~A}, \mathrm{~B}$ ). The accumulation of hydroxyproline content was prevented $(\mathrm{p}<0.01)$ and the myofibroblasts counts were also significantly decreased upon knockdown of TRB3 (figure 5C, D). The antifibrotic effects of TRB3 inhibition were accompanied by inhibition of canonical TGF- $\beta$ signalling. The nuclear 
A
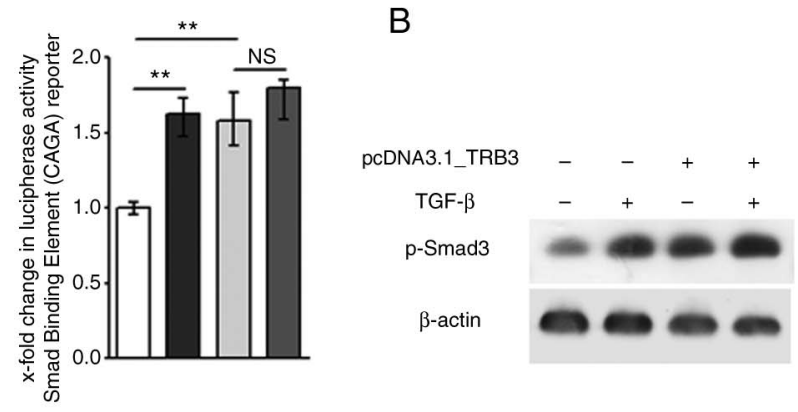

B

$\square$ pcDNA3.1_TRB3
$\mathrm{E}$

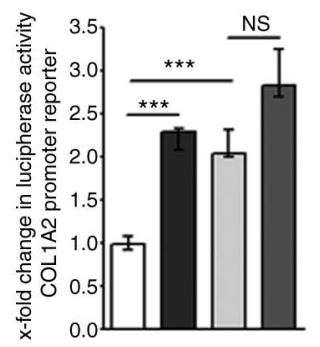

D

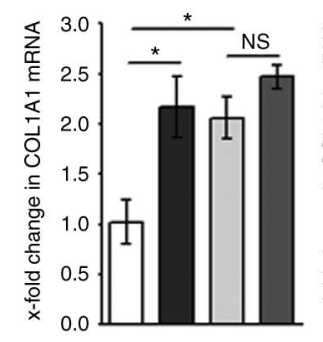

pcDNA3.1 + TGF- $\beta$

pcDNA3.1_TRB3 + TGF- $\beta$
C
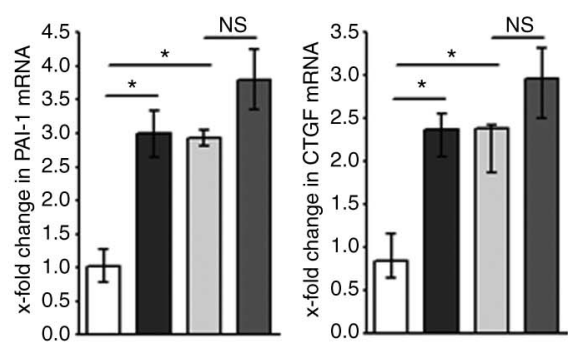

Figure 3 Tribbles homologue 3 (TRB3) induces fibroblast activation and collagen synthesis. (A-C) Overexpression of TRB3 ( $p c D N A 3.1 \_T R B 3$ ) enhances the activity of Smad-dependent CAGA reporter assays $(A ; n=9)$, increases the accumulation of phosphorylated Smad3 $(p-S m a d 3 ; B ; n=4)$ and upregulates the mRNA levels of the prototypical transforming growth factor- $\beta$ (TGF- $\beta$ )/Smad target genes PAl- 1 and CTGF (C for both; $n=6$ for both). (D-F) Overexpression of TRB3 stimulates the activity of COL1A2 reporter assays $(D ; n=9)$, upregulates the mRNA levels of COL1A1 and COL1A2 ( $E$ for both; $n=4$ for both) and enhances the release of collagen into supernatants $(F ; n=4)$. NS, not significant; ${ }^{*} 0.05>p>0.01$; ${ }^{* *} 0.01>\mathrm{p}>0.001 ;{ }^{* * *} \mathrm{p}<0.001$.

accumulation of phosphorylated Smad $2 / 3$ was reduced by $77 \%$ in TRB3-siRNA-treated AdTBR mice compared with shamtreated AdTBR mice ( $\mathrm{p}<0.001$; figure 5E).

\section{Silencing of TRB3 ameliorates bleomycin-induced fibrosis}

To evaluate inhibition of TRB3 in another model with different underlying pathomechanisms, we used a model of bleomycin-induced skin fibrosis. Challenge with bleomycin induced leucocyte infiltration and accumulation of collagen with prominent dermal thickening (figure 6A). In vivo transfection with siRNAs against TRB3 effectively decreased the levels of TRB3 mRNA and protein level in this model (see online supplementary figure S7A, B). Inactivation of TRB3 reduced bleomycin-induced dermal thickening by $50 \%$ compared with bleomycin-challenged mice injected with non-targeting siRNA $(\mathrm{p}<0.05$; figure $6 \mathrm{~A}, \mathrm{~B})$. The hydroxyproline content and the myofibroblasts counts were also significantly decreased upon injection with siRNA against TRB3 (figure 6C, D). The nuclear accumulation of phosphorylated $\mathrm{Smad} 2 / 3$ upon challenge with bleomycin was also reduced by $74 \%$ by inactivation of TRB3 $(\mathrm{p}<0.001$; figure 6E).

\section{DISCUSSION}

We report for the first time an upregulation of TRB3 in fibrosis with prominent overexpression in fibroblasts in lesional SSc skin and in murine models of SSc. Our data demonstrate that the upregulation of TRB3 in fibrotic conditions is caused by TGF- $\beta$. Recombinant TGF- $\beta$ increases the mRNA and protein levels of
TRB3 in cultured fibroblasts and overexpression of a constitutively active TGF- $\beta$ receptor I upregulates TRB 3 in murine skin. We further characterised the intracellular signalling cascades that translate the stimulatory effects of TGF- $\beta$ on TRB3 expression and identified Smad3 as an essential mediator. Fibroblasts expressing TRB3 in SSc skin were uniformly positive for $\mathrm{p}$-Smad $2 / 3$, the central mediators of canonical TGF- $\beta$ signalling. Moreover, knockdown of Smad3 rendered fibroblasts unresponsive to the stimulatory effects of TGF- $\beta$ on TRB3 expression. Consistent with a TGF- $\beta / S$ mad-dependent upregulation of TRB3, TRB3 was also overexpressed in cultured SSc fibroblasts, which are characterised by endogenous activation and persistent TGF- $\beta /$ Smad signalling. ${ }^{1} 4344$ A previous study demonstrated that knockdown of the transcriptional coactivator peroxisome proliferator-activated receptor gamma coactivator (PGC)-1 decreases the expression of TRB 3 in the liver. ${ }^{45}$ As unpublished data from our group indicate that PGC- $1 \alpha$ is required for canonical TGF- $\beta$ signalling, those findings indirectly support the regulatory effects of TGF- $\beta /$ Smad signalling on TRB3.

Our study demonstrates that TRB3 positively regulates canonical TGF- $\beta$ signalling in fibroblasts. Overexpression of TRB3 is sufficient to activate TGF- $\beta$ signalling in fibroblasts, induced accumulation of $\mathrm{p}$-Smad3, activated Smad-dependent transcription with enhanced activity in Smad-dependent reporter assays and increased expression levels of target genes such as PAI-1, CTGF, COL1A1 and COL1A2. In contrast, knockdown of TRB3 severely impaired canonical TGF- $\beta$ signalling and completely prevented Smad-dependent transcription in TGF- $\beta$-stimulated healthy cells. 

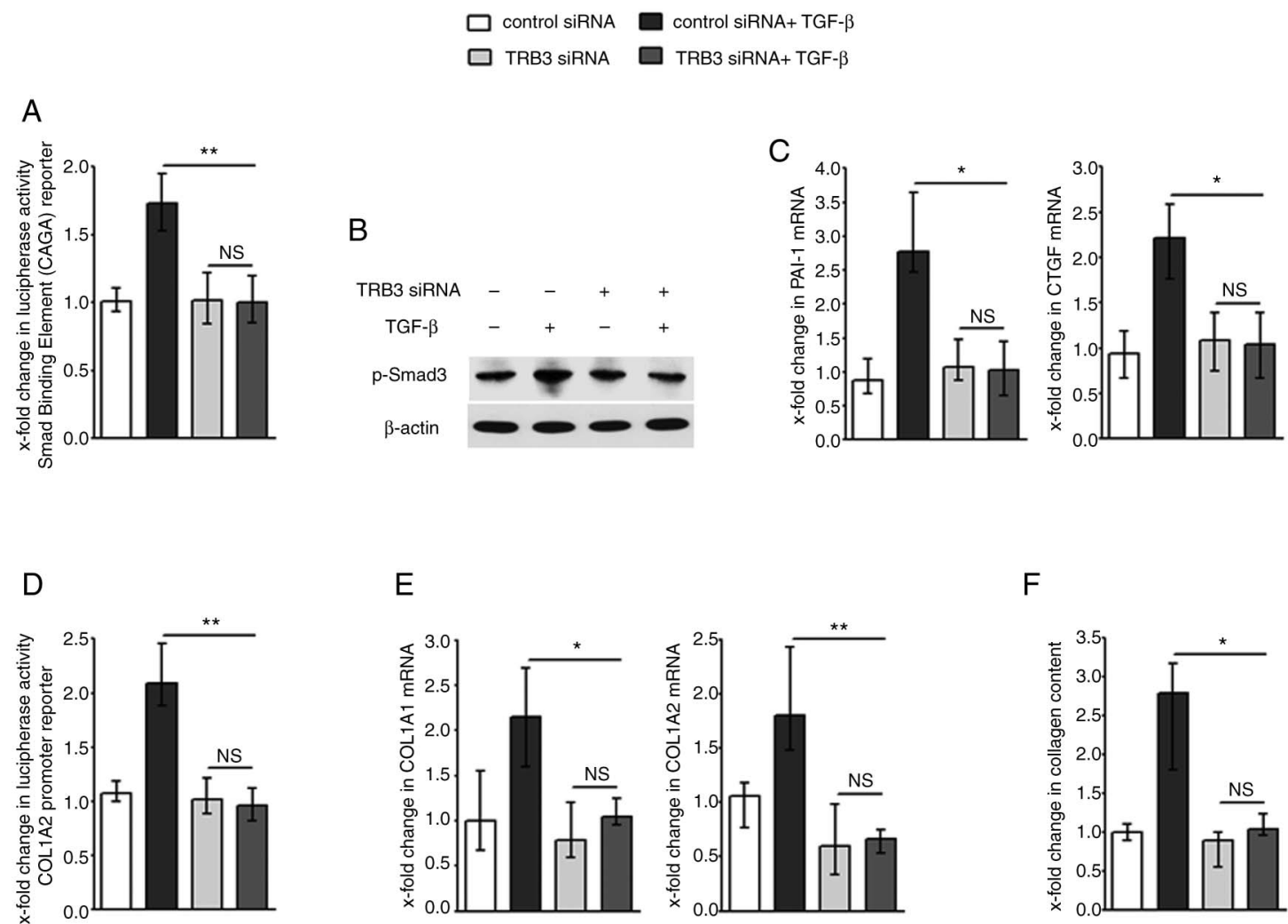

$\mathrm{E}$

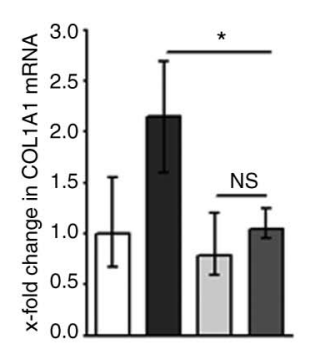

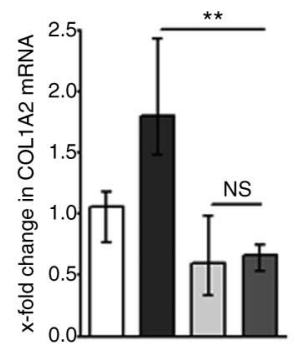

$\mathrm{F}$

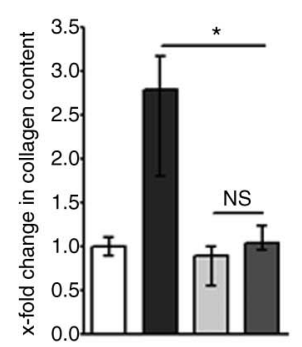

Figure 4 Inhibition of Tribbles homologue 3 (TRB3) prevents transforming growth factor- $\beta$ (TGF- $\beta$ )-induced fibroblast activation. (A-C) Small interfering RNA (siRNA)-mediated knockdown of TRB3 (TRB3 siRNA) inhibits the stimulatory effects of TGF- $\beta$ on Smad binding element/CAGA reporter assays $(A ; n=9)$, on Smad3 phosphorylation ( $p$-Smad3) $(B ; n=4)$ and on PAI-1 and CTGF mRNA ( $C$ for both; $n=6$ for both). (D-F) Knockdown of TRB3 prevents the stimulatory effects of TGF- $\beta$ on COL1A2 reporter activity ( $D ; n=9$ ), COL1A1- and COL1A2 mRNA (E for both; $\mathrm{n}=4$ for both) and on collagen secretion $(\mathrm{F} ; \mathrm{n}=4)$. NS, not significant; ${ }^{*} 0.05>\mathrm{p}>0.01 ;{ }^{* *} 0.01>\mathrm{p}>0.001$.

A

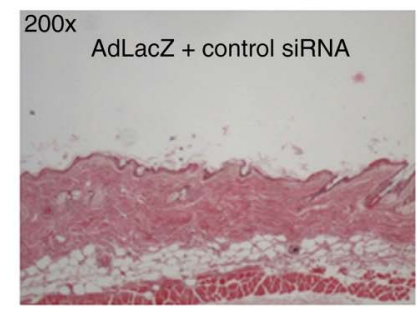

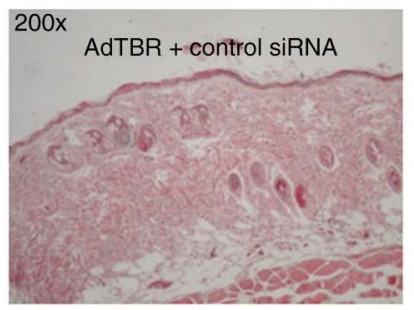

AdTGF + control siRNA

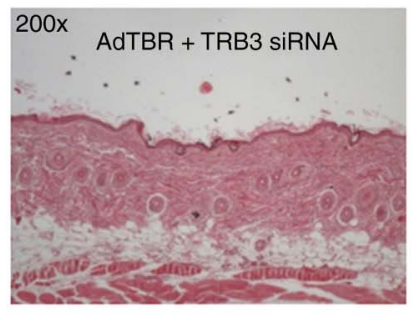

AdTGF + TRB3 siRNA
B

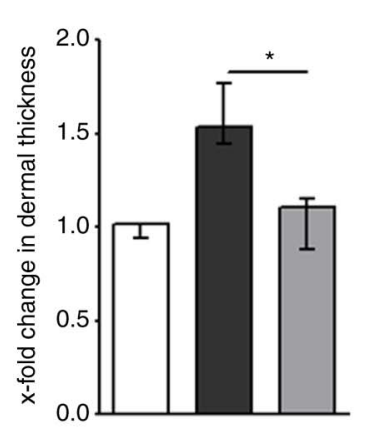

C

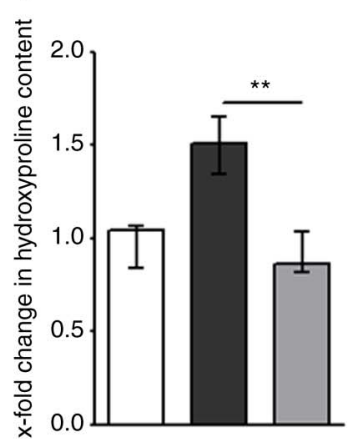

D

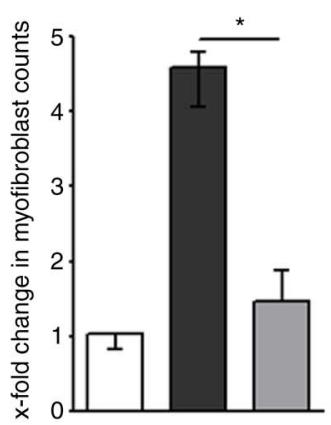

E

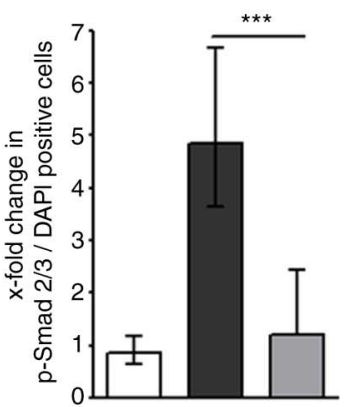

Figure 5 Knockdown of Tribbles homologue 3 (TRB3) protects from transforming growth factor- $\beta$ receptor I (T $\beta R$ I)-induced skin fibrosis. Small interfering RNA (siRNA)-mediated knockdown of TRB3 (TRB3 siRNA) ameliorates T $\beta$ RI-induced skin fibrosis (A) with reduced dermal thickening (B), hydroxyproline content $(C)$, myofibroblasts counts (D) and decreased nuclear accumulation of phosphorylated Smad2/3 ( $p$-Smad2/3) in adenoviral overexpression of a constitutively active T $\beta R I$ (AdTBR) mice injected with TRB3 siRNA as compared with AdTBR mice injected with control siRNA (E). Representative images of $\mathrm{H} \& \mathrm{E}$-stained sections are shown at 200 -fold magnification in $A$ ( $n=6$ per group). ${ }^{*} 0.05>p>0.01 ;{ }^{* *} 0.01>p>0.001$; ${ }^{* * *} \mathrm{p}<0.001$. 

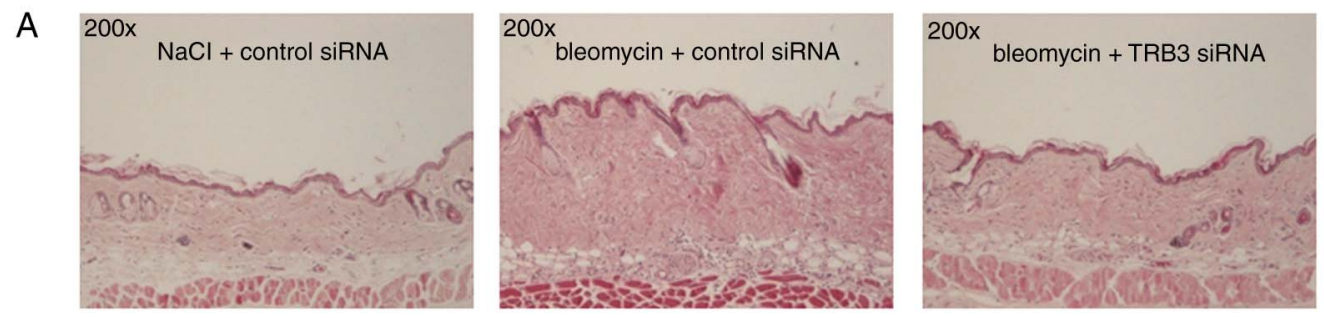

$\square \mathrm{NaCl}+$ control siRNA

$\square$ Bleomycin + control siRNA

Bleomycin + TRB3 siRNA

B

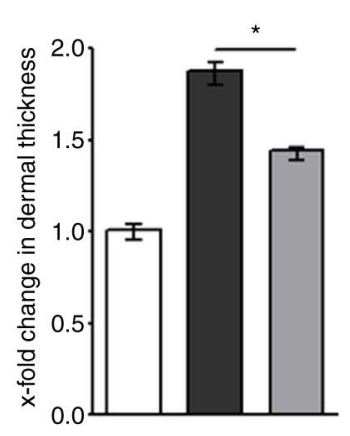

$\mathrm{C}$

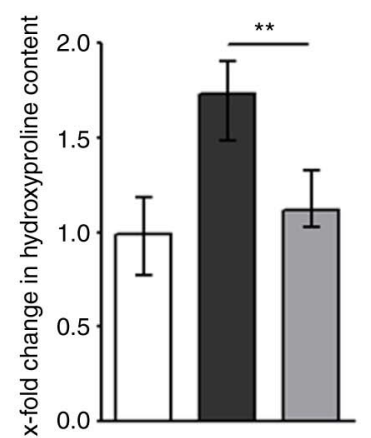

D

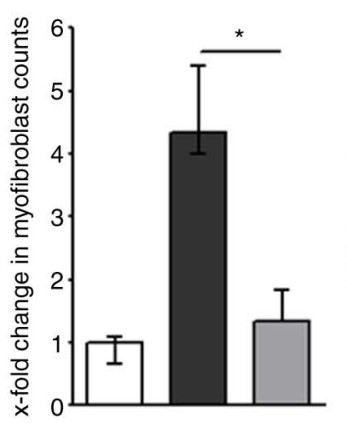

$\mathrm{E}$

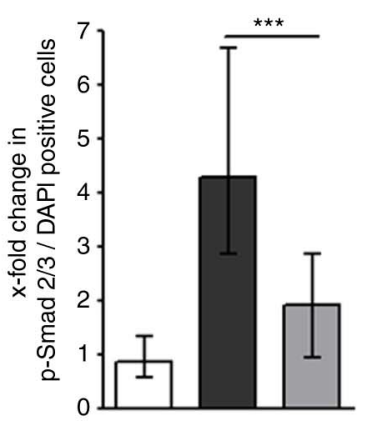

Figure 6 Tribbles homologue 3 (TRB3) knockdown ameliorates bleomycin-induced skin fibrosis. Small interfering RNA (siRNA)-mediated inactivation of TRB3 (TRB3 siRNA) reduces bleomycin-induced skin fibrosis (A) and prevents dermal thickening (B), accumulation of hydroxyproline containing collagen fibres $(C)$, myofibroblast differentiation $(D)$ and inhibits the nuclear accumulation of $p$-Smad2/3 (E). Representative images of H\&E-stained sections are shown at 200 -fold magnification in $A$ ( $n=6$ per group). ${ }^{*} 0.05>p>0.01 ;{ }^{* *} 0.01>p>0.001 ;{ }^{* * *} p<0.001$.

Studies in HEK293T cells suggest that TRB3 can bind to Smad3 and that this interaction may interfere with the nuclear export of Smad3. ${ }^{9}$ Our data show that knockdown of Smad3 abrogates the stimulatory effects of TRB3 overexpression on fibroblasts, indicating that TRB3 modulates fibroblast activation selectively via canonical TGF- $\beta$ signalling. Collectively, these findings demonstrate that TRB3 positively regulates canonical TGF- $\beta$ signalling. As TRB3 is induced by TGF- $\beta$, TRB3 may thus amplify canonical TGF- $\beta$ signalling, thereby contributing to persistently increased TGF- $\beta$ signalling and aberrant fibroblast activation in SSc (see online supplementary figure S8).

Our results also highlight that targeting of TRB3 exerts potent antifibrotic effects in vitro and in vivo. Inactivation of TRB3 prevented the TGF- $\beta$-induced upregulation of type I collagen in cultured healthy fibroblasts and reduced the basal release of collagen in SSc fibroblasts. Moreover, siRNA-mediated knockdown of TRB3 in the skin protected from bleomycin-induced and AdTBR-induced fibrosis and ameliorated skin thickening, collagen accumulation and myofibroblast differentiation in both mouse models. The models used herein mimic different aspects of the pathogenesis of SSc. Bleomycin-induced skin fibrosis mimics inflammatory stages of $\mathrm{SSc}$, in which fibroblast activation is preferentially driven by profibrotic cytokines released by infiltrating leucocytes, whereas AdTBR-induced fibrosis resembles non-inflammatory subgroups of patients, in which progression of fibrosis is driven by endogenous activation of fibroblasts. ${ }^{46}$ Although further studies are needed to support this hypothesis, the potent antifibrotic effects in both models indicate that inactivation of TRB3 may be effective in different subsets and different stages of patients with SSc and may thus be an interesting candidate for targeted therapies.

In summary, we characterise TRB3 as an amplifier of canonical TGF- $\beta$ signalling in SSc. TRB3 is overexpressed in SSc in a
TGF- $\beta$-dependent manner and in turn stimulates canonical TGF- $\beta$ signalling. Silencing of TRB3 abrogates canonical TGF- $\beta$ signalling and ameliorates fibrosis in different preclinical models, further highlighting the central regulatory role of TRB3 on TGF- $\beta$ signalling and fibroblast activation.

Acknowledgements We thank Katja Dreißigacker, Regina Kleinlein and Verena Wäsch for excellent technical assistance.

Contributors Design of the study: MT, LS and JHWD. Acquisition of data: MT, $\mathrm{KP}-\mathrm{Z}, \mathrm{PZ}, \mathrm{BS}, \mathrm{JA}, \mathrm{CD}, \mathrm{AD}$ and $\mathrm{CB}$. Interpretation of data: MT, KP-Z, RB, OD, GS, LS and JHWD. Manuscript preparation: MT, GS, LS and JHWD.

Funding Grants DI 1537/4-1, DI 1537/5-1, DI 1537/7-1, DI 1537/8-1, BE 5191/ 1-1, AK 144/1-1 and SCHE 1583/7-1 of the Deutsche Forschungsgesellschaft, grants A57 of the IZKF in Erlangen, the ELAN-Program of the University of

Erlangen-Nuremberg and the Career Support Award of Medicine of the Ernst Jung Foundation, a Heisenberg Professorship of the Deutsche Forschungsgesellschaft (DI 1537/9-1), the Ministry of Health of the Czech Republic (Research Project No. 00023728) and SVV: 260031.

Competing interests $\mathrm{OD}$ has consultancy relationships and/or has received research funding from Actelion, Pfizer, Ergonex, BMS, Sanofi-Aventis, United BioSource Corporation, medac, Biovitrium, Boehringer Ingelheim, Novartis, 4D Science and Active Biotech in the area of potential treatments of scleroderma; JHWD has consultancy relationships and/or has received research funding from Actelion, Pfizer, Ergonex, BMS, Celgene, Bayer Pharma, Boehringer Ingelheim, JB Therapeutics, Sanofi-Aventis, Novartis, Array Biopharma and Active Biotech in the area of potential treatments of scleroderma and is stock owner of 4D Science GmbH.

Ethics approval Erlangen.

Provenance and peer review Not commissioned; externally peer reviewed.

\section{REFERENCES}

1 Gabrielli A, Avvedimento EV, Krieg T. Scleroderma. N Engl J Med 2009;360:1989-2003.

2 Varga J, Abraham D. Systemic sclerosis: a prototypic multisystem fibrotic disorder. J Clin Invest 2007;117:557-67.

3 Mori Y, Chen SJ, Varga J. Expression and regulation of intracellular SMAD signaling in scleroderma skin fibroblasts. Arthritis Rheum 2003;48:1964-78.

4 Hegedus Z, Czibula A, Kiss-Toth E. Tribbles: novel regulators of cell function; evolutionary aspects. Cell Mol Life Sci 2006;63:1632-41. 
5 Grosshans J, Wieschaus E. A genetic link between morphogenesis and cell division during formation of the ventral furrow in Drosophila. Cell 2000;101:523-31.

6 Boudeau J, Miranda-Saavedra D, Barton GJ, et al. Emerging roles of pseudokinases. Trends Cell Biol 2006;16:443-52.

7 Du K, Herzig S, Kulkarni RN, et al. TRB3: a tribbles homolog that inhibits Akt/PKB activation by insulin in liver. Science 2003;300:1574-7.

8 Ohoka N, Yoshii S, Hattori T, et al. TRB3, a novel ER stress-inducible gene, is induced via ATF4-CHOP pathway and is involved in cell death. EMBO J 2005:24:1243-55.

9 Hua F, Mu R, Liu J, et al. TRB3 interacts with SMAD3 promoting tumor cell migration and invasion. J Cell Sci 2011;124(Pt 19):3235-46.

10 Qi L, Heredia JE, Altarejos JY, et al. TRB3 links the E3 ubiquitin ligase COP1 to lipid metabolism. Science 2006;312:1763-6.

11 Bezy O, Vernochet C, Gesta S, et al. TRB3 blocks adipocyte differentiation through the inhibition of C/EBPbeta transcriptional activity. Mol Cell Biol 2007;27:6818-31.

12 Cravero JD, Carlson CS, Im HJ, et al. Increased expression of the Akt/PKB inhibitor TRB3 in osteoarthritic chondrocytes inhibits insulin-like growth factor 1-mediated cell survival and proteoglycan synthesis. Arthritis Rheum 2009;60:492-500.

13 Bergmann C, Akhmetshina A, Dees C, et al. Inhibition of glycogen synthase kinase 3beta induces dermal fibrosis by activation of the canonical Wnt pathway. Ann Rheum Dis 2011;70:2191-8.

14 Reich N, Maurer B, Akhmetshina A, et al. The transcription factor Fra-2 regulates the production of extracellular matrix in systemic sclerosis. Arthritis Rheum 2010;62:280-90.

15 LeRoy EC, Medsger TA Jr. Criteria for the classification of early systemic sclerosis. J Rheumatol 2001;28:1573-6.

16 van den Hoogen F, Khanna D, Fransen J, et al. 2013 classification criteria for systemic sclerosis: an American college of rheumatology/European league against rheumatism collaborative initiative. Ann Rheum Dis 2013;72:1747-55.

17 Dees C, Tomcik M, Palumbo-Zerr K, et al. JAK-2 as a novel mediator of the profibrotic effects of transforming growth factor beta in systemic sclerosis. Arthritis Rheum 2012;64:3006-15.

18 Palumbo K, Zerr P, Tomcik M, et al. The transcription factor JunD mediates transforming growth factor \{beta\}-induced fibroblast activation and fibrosis in systemic sclerosis. Ann Rheum Dis 2011;70:1320-6.

19 Tomcik M, Palumbo-Zerr K, Zerr P, et al. S100A4 amplifies TGF-beta-induced fibroblast activation in systemic sclerosis. Ann Rheum Dis 2015;74:1748-55.

20 Beyer C, Schramm A, Akhmetshina A, et al. beta-catenin is a central mediator of pro-fibrotic Wnt signaling in systemic sclerosis. Ann Rheum Dis 2012;71: 761-7.

21 Kramer M, Dees C, Huang J, et al. Inhibition of H3K27 histone trimethylation activates fibroblasts and induces fibrosis. Ann Rheum Dis 2013;72:614-20.

22 Tomcik M, Zerr P, Pitkowski J, et al. Heat shock protein 90 (Hsp90) inhibition targets canonical TGF-beta signalling to prevent fibrosis. Ann Rheum Dis 2014;73:1215-22.

23 Avouac J, Palumbo K, Tomcik M, et al. Inhibition of activator protein 1 signaling abrogates transforming growth factor beta-mediated activation of fibroblasts and prevents experimental fibrosis. Arthritis Rheum 2012;64:1642-52.

24 Uhl M, Aulwurm S, Wischhusen J, et al. SD-208, a novel transforming growth factor beta receptor I kinase inhibitor, inhibits growth and invasiveness and enhances immunogenicity of murine and human glioma cells in vitro and in vivo. Cancer Res 2004;64:7954-61.

25 Dees $C$, Tomcik M, Zerr $P$, et al. Notch signalling regulates fibroblast activation and collagen release in systemic sclerosis. Ann Rheum Dis 2011;70:1304-10.
26 Horn A, Palumbo K, Cordazzo C, et al. Hedgehog signaling controls fibroblast activation and tissue fibrosis in systemic sclerosis. Arthritis Rheum 2012;64:2724-33.

27 Lin NY, Beyer C, Giessl A, et al. Autophagy regulates TNFalpha-mediated joint destruction in experimental arthritis. Ann Rheum Dis 2013;72:761-8.

28 Dees $C$, Akhmetshina A, Zerr $P$, et al. Platelet-derived serotonin links vascular disease and tissue fibrosis. J Exp Med. 2011;208:961-72.

29 Dees C, Schlottmann I, Funke R, et al. The Wnt antagonists DKK1 and SFRP1 are downregulated by promoter hypermethylation in systemic sclerosis. Ann Rheum Dis 2014;73:1232-9.

30 Akhmetshina A, Palumbo K, Dees C, et al. Activation of canonical Wnt signalling is required for TGF-beta-mediated fibrosis. Nat Commun. 2012;3:735.

31 Distler JH, Jungel A, Huber LC, et al. Imatinib mesylate reduces production of extracellular matrix and prevents development of experimental dermal fibrosis. Arthritis Rheum 2007;56:311-22.

32 Zerr P, Palumbo-Zerr K, Distler A, et al. Inhibition of hedgehog signaling for the treatment of murine sclerodermatous chronic graft-versus-host disease. Blood 2012;120:2909-17.

33 Horn A, Kireva T, Palumbo-Zerr K, et al. Inhibition of hedgehog signalling prevents experimental fibrosis and induces regression of established fibrosis. Ann Rheum Dis 2012;71:785-9.

34 Khodzhigorova A, Distler A, Lang V, et al. Inhibition of sumoylation prevents experimental fibrosis. Ann Rheum Dis 2012;71:1904-8.

35 Akhmetshina $A$, Venalis $P$, Dees $C$, et al. Treatment with imatinib prevents fibrosis in different preclinical models of systemic sclerosis and induces regression of established fibrosis. Arthritis Rheum 2009;60:219-24.

36 Beyer $\mathrm{C}$, Reichert $\mathrm{H}$, Akan $\mathrm{H}$, et al. Blockade of canonical Wnt signalling ameliorates experimental dermal fibrosis. Ann Rheum Dis 2013;72:1255-8.

37 Dees C, Zerr P, Tomcik M, et al. Inhibition of Notch signaling prevents experimental fibrosis and induces regression of established fibrosis. Arthritis Rheum 2011;63:1396-404

38 Distler A, Deloch L, Huang J, et al. Inactivation of tankyrases reduces experimental fibrosis by inhibiting canonical Wnt signalling. Ann Rheum Dis 2013;72: 1575-80.

39 Palumbo-Zerr K, Horn A, Distler A, et al. Inactivation of fatty acid amide hydrolase exacerbates experimental fibrosis by enhanced endocannabinoid-mediated activation of CB1. Ann Rheum Dis 2012;71:2051-4.

40 Reich N, Tomcik M, Zerr P, et al. Jun N-terminal kinase as a potential molecular target for prevention and treatment of dermal fibrosis. Ann Rheum Dis 2012;71:737-45.

41 Beyer C, Reich N, Schindler SC, et al. Stimulation of soluble guanylate cyclase reduces experimental dermal fibrosis. Ann Rheum Dis 2012;71:1019-26.

42 Beyer $C$, Skapenko A, Distler A, et al. Activation of pregnane $X$ receptor inhibits experimental dermal fibrosis. Ann Rheum Dis 2013;72:621-5.

43 Smith EA, LeRoy EC. A possible role for transforming growth factor-beta in systemic sclerosis. J Invest Dermatol 1990;95(6 Suppl):125S-7S.

44 LeRoy EC. Increased collagen synthesis by scleroderma skin fibroblasts in vitro: a possible defect in the regulation or activation of the scleroderma fibroblast. J Clin Invest 1974;54:880-9.

45 Koo SH, Satoh H, Herzig S, et al. PGC-1 promotes insulin resistance in liver through PPAR-alpha-dependent induction of TRB-3. Nat Med 2004;10:530-4.

46 Beyer C, Schett G, Distler 0, et al. Animal models of systemic sclerosis: prospects and limitations. Arthritis Rheum 2010;62:2831-44. 


\section{ARD Tribbles homologue 3 stimulates canonical TGF- $\beta$ signalling to regulate fibroblast activation and tissue fibrosis}

Michal Tomcik, Katrin Palumbo-Zerr, Pawel Zerr, Barbora Sumova, Jerome Avouac, Clara Dees, Alfiya Distler, Radim Becvar, Oliver Distler, Georg Schett, Ladislav Senolt and Jörg H W Distler

Ann Rheum Dis2016 75: 609-616 originally published online January 20, 2015

doi: 10.1136/annrheumdis-2014-206234

Updated information and services can be found at:

http://ard.bmj.com/content/75/3/609

These include:

Supplementary Material

References

Email alerting service
Supplementary material can be found at:

http://ard.bmj.com/content/suppl/2015/01/20/annrheumdis-2014-2062 34.DC1

This article cites 46 articles, 27 of which you can access for free at: http://ard.bmj.com/content/75/3/609\#ref-list-1

Receive free email alerts when new articles cite this article. Sign up in the box at the top right corner of the online article.

Topic
Collections

Articles on similar topics can be found in the following collections

\section{Notes}

To request permissions go to:

http://group.bmj.com/group/rights-licensing/permissions

To order reprints go to:

http://journals.bmj.com/cgi/reprintform

To subscribe to BMJ go to:

http://group.bmj.com/subscribe/ 